\title{
THE USE OF AUTOGENOUS PARTICULATED DENTIN MIXED WITH PLATELET RICH FIBRIN IN SURGICALLY REMOVED LOWER THIRD MOLAR SOCKET
}

\author{
Abdallah Mahmoud Mohamad Mahmoud*, Mahmoud Ahmad Abdallah ${ }^{* *}$, Atef Mohamad Hassanin ${ }^{* * *}$
}

\begin{abstract}
Objective: The aim of the present study is to evaluate the use of autogenous particulated dentin mixed with platelet rich fibrin in surgically removed lower third molar socket. Materials and Methods: Sixteen patients ( 7 male and 9 female) were enrolled in the study. Their age ranged from 20 to 37 years. Patients were randomly divided into 2 groups. Group I, PD/PRF mix was grafted into the extraction socket. Group II, extraction socket was left without grafting. The CD, CAL and WAG of lower second molar were measured at 2, 4, 8 and 12weekes after surgery. MBL at the distal surface of lower second molar was evaluated at 1 and 6 months after surgery. BD was measured at 6 month after surgery. Results: The PD/PRF mix group showed significant reduction of $\mathrm{CD}$ at the distal of lower second molar than the no grafted group. The PD/PRF mix group showed significant reduction of CAL at the distal of lower second molar than the no grafted group. The PD/PRF mix group showed significant less reduction of WAG at the distal of lower second molar than the no grafted group. The PD/PRF mix group showed significant less amount of MBL loss at the distal of lower second molar than the no grafted group. There was a significant increase of $\mathrm{BD}$ at the distal of lower second molar at 6 month in PD/PRF mix group than the no grafted group. Conclusion: Autogenous particulate dentin and platelet rich fibrin mix provided promising clinical outcomes and considered an ideal bioactive graft material for hard tissue regeneration. Autogenous particulate dentin and platelet rich fibrin mix assist healing and counteract pronounced resorption of alveolar process at the distal surface of second molar with preservation of the periodontium.
\end{abstract}

KEYWORDS: Autogenous Particulated Dentin, Platelet Rich Fibrin, Surgically Removed Lower Third Molar.

\section{INTRODUCTION}

Surgical removal of the impacted lower $3^{\text {rd }}$ molars is one of the most common procedures in the oral surgery ${ }^{(1)}$. This may induce significant dimensional changes of the alveolar bone and produce large bony defect distal to lower $2^{\text {nd }}$ molar.
This bony defect lead to formation of periodontal pocket, accumulation of the food, bad odor and pain distal to the lower $2^{\text {rd }}$ molar ${ }^{(2)}$. Several methods have been used to overcome the problem of pocket formation at the distal of lower $2^{\text {nd }}$ molar such as modification of flap design and some new surgical techniques as orthodontic-assisted extraction of

* Masters Candidate, Department of Oral and Maxillofacial Surgery, Faculty of Dental Medicine, (Boys, Cairo), Al-Azhar University

** Professor, Department of Oral and Maxillofacial Surgery, Faculty of Dental Medicine, (Boys, Cairo), Al-Azhar University

*** Associate Professor, Department of Oral and Maxillofacial Surgery, Faculty of Dental Medicine, (Boys, Cairo), Al-Azhar University.

-Corresponding author: amar.ali3721648@gmail.com

DOI: 10.21608 /ajdsm.2020.28393.1053 
impacted third molars, pericoronal ostectomy and grafting ${ }^{(3-5)}$.

Fresh autogenous bone graft is still considered the gold standard since it exhibits bioactive cell instructive matrix properties and is non-immunogenic and non - pathogenic in spite of the need for harvesting bone and possible morbidity resulting from it. Due to the drawback of autogenous grafting, allogeneic bone and synthetic mineral materials are used nowadays for grafting in jaw bony defect ${ }^{(6-8)}$.

It has been reported that extracted teeth from patients that undergo a process of cleaning, grinding, demineralization and sterilization is a very effective graft to fill alveolar bone defects of same patient. In a recent study, the dentin particles were immediately filled into the alveolar socket after the extraction; soft tissue closure was performed with a free gingival graft of the palate. Four months later, implant was placed in the augmented area, which osseointegrated successfully and could be restored prosthodontically ${ }^{(9-13)}$.

Due to the hard nature of particulated dentine, it is thought that platelet rich fibrin may play a crucial role in making the graft pasty. Platelet rich fibrin has an important role in periodontal regeneration as they are reservoirs of growth factors and cytokines which are the key factors for regeneration of the bone and maturation of the soft tissue ${ }^{(14)}$. In this study, it was assumed that placement of autogenous particulated dentin and PRF mix in extraction socket of lower $3^{\text {rd }}$ molars might improve healing and prevent periodontal pocketing distal to lower $2^{\text {rd }}$ molar.

\section{MATERIALS AND METHODS}

Sixteen patients ( 7 male and 9 female) were enrolled in the study. Their age ranged from 20 to 37 years. Inclusion criteria: Partially impacted mandibular $3^{\text {rd }}$ molar, age ranges between $18-40$ years, and the presence of adjacent lower second molar tooth.

Exclusion criteria: Uncontrolled diabetic patient, patient having bleeding disorders, presence of active infection at the site of surgery, presence of any pathologic lesion at the site of surgery, patients with history of radiotherapy or chemotherapy.

Patients were randomly divided into 2 groups. Group I, PD/PRF mix was grafted into the extraction socket. Group II, extraction socket was left without grafting. The CD, CAL and WAG of lower second molar were measured at 2, 4, 8 and 12 weekes after surgery. MBL at the distal surface of lower second molar was evaluated at 1 and 6months after surgery. BD was measured at 6 month after surgery, Fig (1).

\section{RESULTS}

The PD/PRF mix group showed significant reduction of $\mathrm{CD}$ at the distal of lower second molar than the no grafted group. The PD/PRF mix group showed significant reduction of CAL at the distal of lower second molar than the no grafted group. The PD/PRF mix group showed significant less reduction of WAG at the distal of lower second molar than the no grafted group. The PD/PRF mix group showed significant less amount of MBL loss at the distal of lower second molar than the no grafted group. There was a significant increase of $\mathrm{BD}$ at the distal of lower second molar at 6 month in PD/PRF mix group than the no grafted group. 


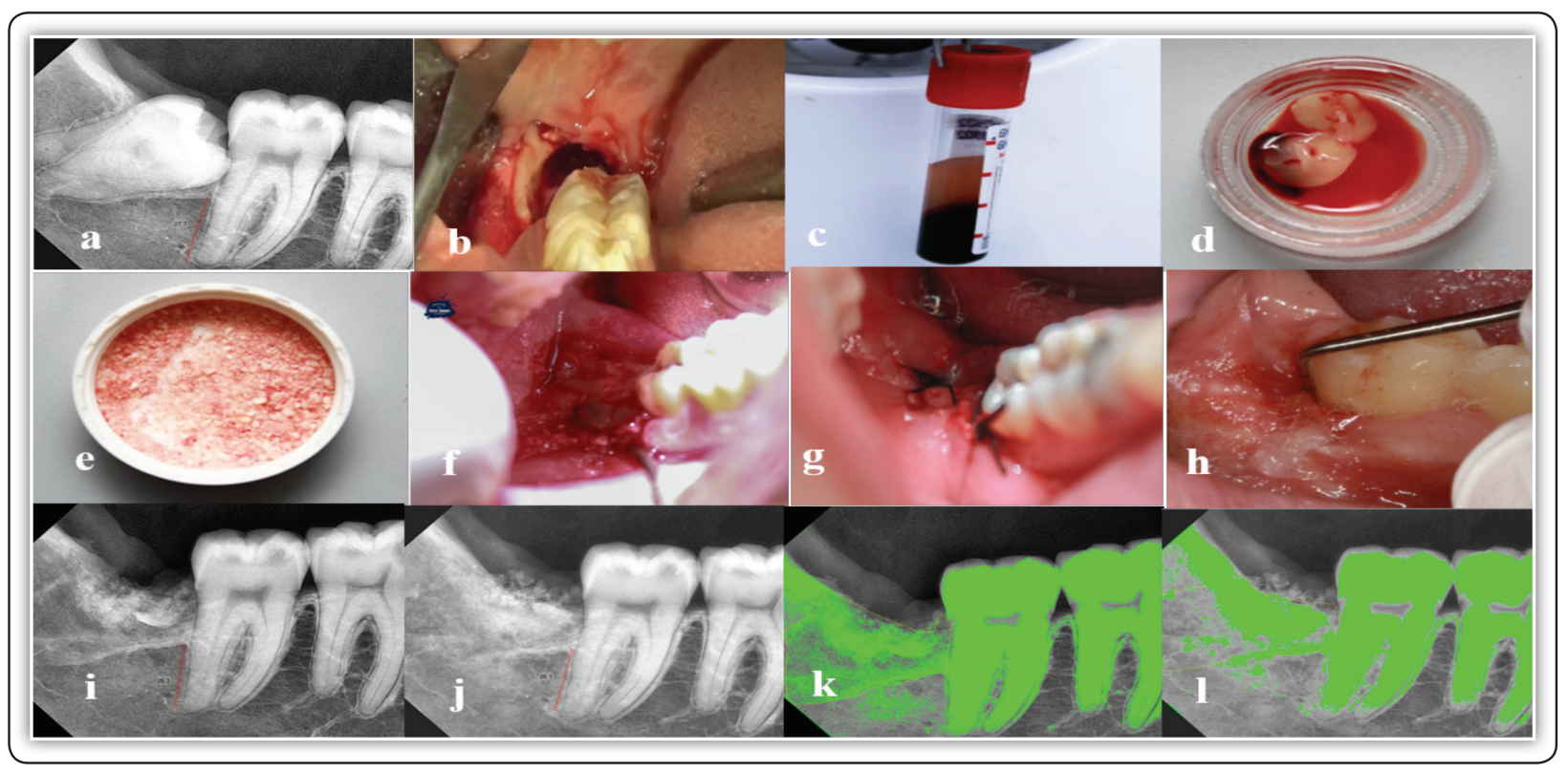

FIG (1) a; Pre-operative marginal bone measurement, b; Socket immediately after extraction, c; Blood sample after centrifugation for 10 minutes, d; PRF membrane, e; APD ready for grafting, f; APD and PRF mix placed in extracted socket, $\mathbf{g}$; sutured wound, $\mathbf{h}$; postoperative measurement of crevicular depth with periodontal probe, $\mathbf{i}$; Measurement of MBL one month after surgery, $\mathbf{j}$; Measurement of MBL 6 month after surgery, k; Measurement of BD immediately after surgery, l; Measurement of BD 6 month after surgery.

TABLE (1): Comparison between the two studied groups according to mean Crevicular depth, Clinical attachment level (mm), Width of attached gingiva (mm), Marginal bone loss (mm), and Bone density.

\begin{tabular}{|c|c|c|c|c|c|}
\hline & \multicolumn{2}{|c|}{ Group I } & \multicolumn{2}{|c|}{ Group II } & \multirow{2}{*}{$\boldsymbol{P}$} \\
\hline & Mean & \pm SD & Mean & \pm SD & \\
\hline \multicolumn{6}{|c|}{ Crevicular depth } \\
\hline Before & 7.56 & 1.07 & 7.13 & 0.83 & 0.701 \\
\hline 2 Weekes & 7.31 & 0.78 & 7.25 & 0.65 & 0.399 \\
\hline 4 Weekes & 7.25 & 0.65 & 7.31 & 0.65 & 0.851 \\
\hline 8 Weekes & 6.75 & 0.65 & 7.56 & 0.56 & $0.001^{*}$ \\
\hline 12 Weekes & 6.15 & 0.75 & 7.75 & 0.65 & $<0.001^{*}$ \\
\hline \multicolumn{6}{|c|}{$\begin{array}{l}\text { Clinical attachment level } \\
(\mathrm{mm})\end{array}$} \\
\hline Before & 5.44 & 0.90 & 6.13 & 0.83 & 0.136 \\
\hline 2 Weekes & 5.44 & 0.90 & 6.25 & 0.65 & 0.059 \\
\hline 4 Weekes & 5.13 & 0.83 & 6.31 & 0.65 & $0.007^{*}$ \\
\hline 8 Weekes & 4.69 & 0.75 & 6.56 & 0.56 & $<0.001^{*}$ \\
\hline 12 Weekes & 4.0 & 0.46 & 6.75 & 0.65 & $<0.001^{*}$ \\
\hline \multicolumn{6}{|c|}{$\begin{array}{l}\text { Width of attached gingiva } \\
\text { (mm) }\end{array}$} \\
\hline Before & 4.44 & 0.90 & 4.13 & 0.86 & 0.277 \\
\hline 2 Weekes & 4.44 & 0.90 & 4.0 & 0.53 & 0.263 \\
\hline 4 Weekes & 4.23 & 0.83 & 3.94 & 0.83 & 1.000 \\
\hline 8 Weekes & 4.21 & 0.59 & 3.50 & 0.60 & $0.008^{*}$ \\
\hline 12 Weekes & 4.11 & 0.53 & 3.31 & 0.46 & $0.006^{*}$ \\
\hline \multicolumn{6}{|c|}{ Marginal bone loss ( $\mathrm{mm}$ ) } \\
\hline Before & 27.10 & 0.64 & 27.30 & 0.64 & 0.543 \\
\hline 1 month & 26.43 & 0.64 & 25.75 & 0.72 & 0.067 \\
\hline 6 months & 26.31 & 0.55 & 24.98 & 0.74 & $0.012^{*}$ \\
\hline \multicolumn{6}{|c|}{ Bone density } \\
\hline Immediate & 182.14 & 47.09 & 139.29 & 45.67 & 0.110 \\
\hline 6 months & 224.14 & 47.69 & 178.43 & 37.26 & $0.008^{*}$ \\
\hline
\end{tabular}

$p: p$ value for comparing between the two studied groups *: Statistically significant at $p \leq 0.05$ 


\section{DISCUSSION}

There is a consensus that PRF significantly reduced postoperative pain, trismus, swelling and dry socket after mandibular third molar extraction. In spite of the great advantages of PRF in healing process, PRF single use seems to have no beneficial role in bone healing and have no effect on the periodontal status of the lower second molars after extraction of the mandibular third molars. The findings of Zahid and Nadershah (15) study demonstrated that A-PRF successfully act as a potential biomaterial for lessening the severity of pain and swelling after third molar surgery. They concluded that long-term trials with a larger sample size and more methodically sound assessment tools are needed to obtain more meaningful results on periodontal regeneration. Xiang et al ${ }^{(16)}$ confirmed the beneficial effect of PRF in relieving pain and swelling and reducing the incidence of alveolar osteitis after the extraction of an impacted lower third molar. However, they found no difference with respect to trismus, osteoblastic activity, and soft tissue healing. Girish Rao et al ${ }^{(17)}$ found a definite improvement in the regeneration of bone after third molar surgery in cases treated with PRF as compared to the control group postoperatively. The enhanced bone density increase is because of the three most important growth factors from the PRF are PDGF, insulin-like growth factor-I, and transforming growth factor- $\beta$ (TGF- $\beta$ ). Varghese et al (18) investigated the potential of platelet-rich fibrin (PRF) for osseous regeneration and soft tissue healing in mandibular third molar impaction sockets. They concluded that there was evidence for better osseous regeneration and soft tissue healing in response to PRF.

In the current study, we have used a mix of two autologous biomaterials: particulate dentin and PRF. Particulate dentin was mixed with PRF membrane for two reasons; the first is to give the particulate dentin a pasty homogenous consistency to facilitate graft manipulation and the second is to benefit of the great regenerative potential of both PD and PRF. Particulate dentin was prepared from the freshly extracted lower third molar tooth with the use of Smart Dentin Grinder. Smart Dentin Grinder facilitates preparation of autogenous particulate dentin graft in as little as 15 min during a single surgical session ${ }^{(12)}$. The ability of particulate dentin to promote bone formation in the lower third molar sockets can be explained by its common embryological origin and similar composition. PRF is the second generation of platelet concentrate obtained by a simple protocol of centrifugation (3000 rpm during $10 \mathrm{~min}$ ) without additives. This straightforward and inexpensive technology has shown to be able to enhance the healing process and to promote tissue regeneration due to the presence of platelets and leukocytes which release growth factors and cytokines involved in the healing process ${ }^{(19)}$.

The incorporation of small PRF pieces in the particulate dentin to reduce the amount of particulate graft seems to maximize the regenerative process. The slow release of growth factors from PRF and their fibrin mesh gives an excellent scaffold for the migration of stem cells and osteogenic cells, possibly improving the angiogenesis and the new bone formation. The 1:1 ratio of dentin and PRF in the particulate dentin produces a unique tridimensional distribution, generating spaces between the dentin particles so that the angiogenesis and cell migration can be facilitated ${ }^{(20)}$. It was found that using a mixture of autogenous particulate dentin and PRF for grafting of extraction sockets, provides effective socket preservation, additionally sufficient and qualified new bone formation in the grafted sites ${ }^{(21)}$.

Results of the present study indicated that the particulate dentin and platelet rich fibrin PD/PRF mix group have significant improvement of the periodontal status at the distal of lower second molars after extraction of impacted lower third molars than do the no grafted group. The preoperative mean crevicular depth $\mathrm{CD}$ in PD/PRF mix group was 7.56 
$\mathrm{mm}$, while at 8 and 12 weeks postoperatively, the mean crevicular depth was reduced significantly to $6.75 \mathrm{~mm}$ and $6.15 \mathrm{~mm}$ respectively. Slight but significant increase of the mean CD was seen in no grafted group at 12 week postoperatively.

The preoperative mean clinical attachment level $\mathrm{CAL}$ in PD/PRF mix group was $5.44 \mathrm{~mm}$, while at 8 and 12 weeks postoperatively, the mean CAL was reduced significantly to $4.69 \mathrm{~mm}$ and $4.0 \mathrm{~mm}$ respectively. The reduction in CAL mean value means attachment level gain. Slight but significant increase of the mean CAL was seen in no grafted group at 12 week postoperatively. The increase in CAL mean value means that there is a loss in the attachment level. The preoperative mean width of attached gingiva WAG in PD/PRF mix group was $4.44 \mathrm{~mm}$, by the 12th week it became $4.11 \mathrm{~mm}$. while in no grafted group it was $4.13 \mathrm{~mm}$ before surgery and became $3.31 \mathrm{~mm}$ at the 12 th week. The PD/PRF mix group showed significant lesser reduction in the mean WAG than the no grafted group.

The improvement of the periodontal status at the distal of lower second molars after extraction of impacted lower third molars in grafted group could be attributed to the viability of $\mathrm{PD} / \mathrm{PRF}$ mix and its regenerative ability. The PD promotes bone formation due to its common embryological origin and similar composition ${ }^{27)}$. PRF is able to enhance the healing process and to promote tissue regeneration due to the action of growth factors and cytokines involved in the healing process ${ }^{(19)}$.

Results of the present study is in accordance with that of many investigators (23-26) who found that grafting the distal to mandibular second molars resulted in a significant reduction in the probing pocket depth and clinical attachment level gain and in disagreement with Tabrizi et al ${ }^{(27)}$ and Zahid and Nadershah ${ }^{(15)}$ who found no difference in PD and CAL between the grafted and no grafted groups.

The marginal bone level MBL was significantly reduced in both groups. However, the PD/PRF mix group significantly has less amount of MBL reduc- tion than do the no grafted group. This is because the particulate dentin improves bone remodeling capabilities, providing a physical matrix for the deposition of new bone and thereby preserves the height of bone crests ${ }^{(22)}$. The slow release of growth factors from PRF and their fibrin mesh gives an excellent scaffold for the migration of stem cells and osteogenic cells, possibly improving the angiogenesis and the new bone formation ${ }^{(19)}$.

The grafted PD/PRF mix was followed by radiographic observations. The biomaterial was readily identifiable on X-ray, being much denser than the adjacent bone ${ }^{21}$. Radiographic evaluation showed noticeable enhancement of radiopacity on the grafted socket by the autogenous PD/PRF. Regarding the density measurements, the bone density mean value was increased in both groups at 6month; however, this increase was nonsignificant. The mean of the $\mathrm{PD} / \mathrm{PRF}$ mix group remained, significantly, higher than that of the no grafted group after 6 months.

\section{CONCLUSION}

Autogenous particulate dentin and platelet rich fibrin mix provided promising clinical outcomes and considered an ideal bioactive graft material for hard tissue regeneration. Autogenous particulate dentin and platelet rich fibrin mix assist healing and counteract pronounced resorption of alveolar process at the distal surface of second molar with preservation of the periodontium.

\section{REFERENCES}

1. Yuasa H, Sugiura M. Clinical postoperative findings after removal of impacted mandibular third molars: Prediction of postoperative facial swelling and pain based on preoperative variables. Br J Oral Maxillofac Surg. 2004; 42:209-14.

2. Horowitz R, Holtzclaw D, Rosen PS A review on alveolar ridge preservation following tooth extraction. J Evid Based Dent Pract .2012; 12: 149-60.

3. Gallesio C, Berrone M, Ruga E, Boffano P. Surgical extraction of impacted inferior third molars at risk for inferior alveolar nerve injury. J Craniofac Surg. 2010;2:2003-7. 
4. Alessandri G, Bendandi M, Laino L, Checchi V, Checchi L. Orthodontic extraction: riskless extraction of impacted lower third molars close to the mandibular canal. J Oral Maxillofac Surg. 2007; 65:2580-86.

5. Tolstunov L, Javid B, Keyes L, Nattestad A. Pericoronal ostectomy: an alternative surgical technique for management of mandibular third molars in close proximity to the inferior alveolar nerve. J Oral Maxillofac Surg 2011; 69:1858-66.

6. Qin C, Brunn J, Cadena E, Ridall A, Tsujigiwa H. The expression of dentin sialophosphoprotein gene in bone. $\mathrm{J}$ Dent Res .2014; 81: 392-94.

7. Fillingham Y, Jacobs J. Bone grafts and their substitutes. Bone Joint J. 2016; 98:6-9.

8. Fernández R, Bucchi C, Navarro P, Beltrán V, Borie E. Bone grafts utilized in dentistry: an analysis of patients' preferences. MC Med Ethics. 2015; 20: 16-71.

9. Kim Y, Kim S, Byeon J, Lee H, Um I. Development of a novel bone grafting material using autogenous teeth. Oral Surg Oral Med Oral Pathol Oral Radiol Endod .2010; 109: 496-503.

10. Murata M, Akazawa T, Mitsugi M. Human dentin as novel biomaterial for bone regeneration, biomaterials - physics and chemistry, Rosario Pignatello , 2011 ; P127-40.

11. Qin X, Raj R, Liao X, Shi W, Ma B. Using rigidly fixed autogenous Tooth graft to repair bone defect: an animal model. Dent Traumatol. 2014; 30: 380-84.

12. Binderman I, Hallel G, Nardy C, Yaffe A, and Sapoznikov L. A Novel Procedure to Process Extracted Teeth for Immediate Grafting of Autogenous Dentin. J.Interdiscipl Med Dent Sci, 2014; 2(6): 1000154.

13. Valdec S, Pasic P, Soltermann A, Thoma D, Stadlinger B, Rücker M. Alveolar ridge preservation with autologous particulated dentin, Int J Implant Dent. 2017; 3-12.

14. Aroca S, Keglevich T, Barbieri B, Gera.I, Etienne D. Clinical evaluation of a modified coronally advanced flap alone or in combination with a platelet-rich fibrin membrane for the treatment of adjacent multiple gingival recessions: A 6-month study J Periodontol, 2009;80: 244-52.

15. Zahid TM, Nadershah M. Effect of Advanced Plateletrich Fibrin on Wound Healing After Third Molar Extraction: A Split-mouth Randomized Double-blind Study.J Contemp Dent Pract. 2019; 20 :1164-70.

16. Xiang X, Shi P, Zhang P, Shen J, Kang J. Impact of Platelet-Rich Fibrin on Mandibular Third Molar Surgery Recovery: A Systematic Review and Meta-Analysis. BMC Oral Health 2019; 19:163.
17. Girish Rao S, Bhat P, Nagesh KS, Rao GH, Mirle B, Kharbhari L et al. Bone regeneration in extraction sockets with autologous platelet rich fibrin gel. J Maxillofac Oral Surg. 2013; 12:11-6.

18. Varghese MP, Manuel S, Kumar L K S. Potential for Osseous Regeneration of Platelet-Rich Fibrin-A Comparative Study in Mandibular Third Molar Impaction Sockets. J Oral Maxillofac Surg. 2017;75:1322-9.

19. Dohan DM, Choukroun J, Diss A, Dohan SL, Dohan AJ, Mouhyi J and Gogly B. Platelet-rich fibrin (PRF): a second-generation platelet concentrate.Part-1 :technological concepts and evolution. Oral surg Oral med Oral Pathol Oral Radiol Endod. 2006; 101: e37-44.

20. Andrade C, Camino J , Nally M ,Quirynen M ,Martínez B , Pinto N. Combining autologous particulate dentin, L-PRF, and fibrinogen to create a matrix for predictable ridge preservation: a pilot clinical study. Clin Oral Investig. 2020;24:1151- 60 .

21. Yuceer E, Ozkan N, Emin M. Socket preservation using autogenous particulated dentin graft - a clinical study. Clin Oral Impl Res. 2018;29.

22. Nadershah M, Zahid T. Use of Autogenous Dentin Graft in Mandibular Third Molar Extraction Sockets: A SplitMouth Randomized Double-Blind Study. Int.J. Pharm. Res. Allied Sci., 2019; 8:73-79.

23. Hassan K, Marei H, Alagl A. Does Grafting of Third Molar Extraction Sockets Enhance Periodontal Measures in 30- To 35-year-old Patients? J Oral Maxillofac Surg 2012; 70:757-64.

24. Sammartino G, Tia M, Bucci T, Wang HL. Prevention of Mandibular Third Molar Extraction-Associated Periodontal Defects: A Comparative Study. J Periodontol 2009; 80:389-96.

25. Singh M, Bhate K, Kulkarni D, Kumar SN, Kathariya R. The Effect of Alloplastic Bone Graft and Absorbable Gelatin Sponge in Prevention of Periodontal Defects on the Distal Aspect of Mandibular Second Molars, After Surgical Removal of Impacted Mandibular Third Molar: A Comparative Prospective Study. J Maxillofac Oral Surg. 2015; 14: 101-06.

26. Kuperschlag A, Keršytė G, Kurtzman G M, Horowitz R A. Autogenous Dentin Grafting of Osseous Defects Distal to Mandibular Second Molars After Extraction of Impacted Third Molars. Compend Contin Educ Dent, 2020; 41: 76-82.

27. Tabrizi R, Khorshidi H, Shahidi S, Gholami M, Kalbasi $\mathrm{S}$ and Khayati A. Use of lincomycin-impregnated demineralized freeze-dried bone allograft in the periodontal defect after third molar surgery. J Oral Maxillofac Surg. 2014;72:850-7. 